\title{
Simple analytic tool for spectral control of dipole plasmon resonance frequency for gold and silver nanoparticles
}

\author{
Anastasiya Derkachova, Krystyna Kolwas \\ Institute of Physics, Polish Academy of Sciences, Al. Lotników 32/46, Warszawa
}

Received May 14, 2013; accepted June 27, 2013; published June 30, 2013

\begin{abstract}
We give a mathematically simple tool allowing to predict dipole localized surface plasmon resonance frequency as a function of size for gold and silver nanoparticles in water. We compare the results with our previous general study resulting from strict solutions of the eigenvalue problem of metal nanospheres embedded in various dielectrics.
\end{abstract}

The excitation of localized surface plasmons (LSP) on metal nanospheres offers a variety of applications in nanotechnology, biophysics, biochemistry etc. One of the most attractive advantages of LSP's application is the local enhancement of the electromagnetic (EM) field in the very proximity of the nanosphere. This effect takes place when the frequency of an incoming light field corresponds to the characteristic resonance frequency of LSP. This property is applied in surface-enhanced Raman spectroscopy (SERS), high-resolution microscopy, improvement of plasmonic solar cells and many plasmonic applications such as non-diffraction-limited guiding of light (e.g. via a chain of gold nanospheres). Gold and silver nanoparticles are important components of many plasmonic devices due to their chemical inertness and unique optical properties in the visible and near-infrared spectral range. In contrast to a metal with a flat surface, the curved surface of a nanoparticle enables direct optical excitation of plasmon resonances. It has been demonstrated that the plasmon-related optical features are sensitive to the size, shape, and environment of nanoparticles. Such sensitivity permits to tune the optical response of nanoparticles, thus making them suitable for a wide range of applications in photonics and optoelectronics. The possibility of manipulating LSP frequency is very attractive in applications.

Effective controlling of spectral properties of plasmonic nanospheres is not possible without knowing the direct dependence of LSP resonance frequency and resonance spectral width (defined by plasmon damping rates) on particle size.

The solutions of Mie deliver indispensable formalism enabling to calculate the EM fields in the near and far field regions around a spherical particle illuminated by a plane monochromatic wave. The resulting light intensities as well as the absorption and extinction cross-sections can be found for chosen particle size. Mie theory supposes that the nanoparticles and the surrounding medium are homogeneous and characterized by bulk permittivity functions which are the outside parameters of the theory. The problem is solved in spherical coordinates where electromagnetic fields are expressed as infinite sums of the partial electromagnetic waves of the "electric" (or transverse magnetic (TM)) and "magnetic" (or transverse electric (TE)) type.

Usually, the positions of successive peaks, appearing in the extinction or absorption spectra are interpreted as directly related to positions of surface plasmon resonances. The spectra collected for nanoparticles of different sizes have been used as a source of experimental data allowing to reconstruct the dependence of the (mostly dipole only) resonance position on size (e.g. [1$3])$. In [3, 4] we discuss some problems arising from such an interpretation in more details and the reasons why Mie theory is not a handy tool in studying the size dependence of plasmon resonance frequencies. In particular, one of disadvantages is that the spectra must be collected for nanoparticles of many different sizes, which is the condition for a reliable reconstruct of such size dependence.

In [3-7] we gave the direct and strict size characterisation of LSP resonances by solving the eigenvalue problem of nanospheres of different metals embedded in some dielectric environments. We found the radius dependence of resonance frequencies of multipolar LSP modes and the corresponding damping rates in alkali and noble nanoparticles and we increased the size range for data for the particles as large as 1000nm [7]. Such direct LSP size characteristics allow to overcome the inconveniences of the approach based on measuring the positions of maxima in the spectra treated as the indication of LSP resonance. The eigenvalue problem for the nanosphere was formulated in the absence of external, incoming fields, in analogy to the description of surface plasmons (SPP) at a flat metal-dielectric interface. The dispersion relation for SPP propagating along the flat interface between the metal-dielectric boundary can be expressed by a simple analytic function:

$$
k_{S P P}(\omega)=\frac{\omega}{c} \sqrt{\frac{\varepsilon_{m}(\omega) \cdot \varepsilon_{d}}{\varepsilon_{m}(\omega)+\varepsilon_{d}}},
$$

where $\varepsilon_{m}(\omega)$ is the dielectric function of a metal and $\varepsilon_{d}$ of a dielectric. However, in the case of the spherical metaldielectric boundary, there is no direct connection of the 
LSP wave vector to the frequency of the electric field. Resonant frequencies of plasmon oscillations can be found numerically by solving the dispersion relation for EM field at the sphere boundary:

$$
\begin{aligned}
& \sqrt{\sqrt{\varepsilon_{m}(\omega)}} \xi_{l}\left(k_{d}(\omega) R\right) \psi_{l}^{\prime}\left(k_{m}(\omega) R\right)+ \\
& -\sqrt{\varepsilon_{d}} \xi_{l}^{\prime}\left(k_{d}(\omega) R\right) \psi_{l}\left(k_{m}(\omega) R\right)=0,
\end{aligned}
$$

where $k_{m}(\omega)=(\omega / c) \sqrt{\varepsilon_{m}(\omega)}$ and $k_{d}(\omega)=(\omega / c) \sqrt{\varepsilon_{d}}$ are the wave numbers of the light field inside and outside the sphere. Solutions of Eq. (2) exist for complex frequencies of EM field only: $\Omega(R)=\omega_{l}^{\prime}(R)+i \omega_{l}^{\prime \prime}(R), l=1,2,3, \ldots(l=1$ for the dipole mode), where $\omega_{l}^{\prime}(R)$ are the frequencies of oscillations of the EM surface plasmon modes expressed in $\mathrm{eV}$ and the negative $\omega_{l}{ }^{\prime \prime}(R)$ define the damping times $T_{l}(R)=2 \pi \hbar /\left|\omega_{l}{ }^{\prime \prime}(R)\right|$ of plasmon oscillations due to the absorption of radiation by the metal and LSP radiative losses $[4,7]$.

Noble metal nanospheres like gold and silver are widely used in nanotechnology, biophysics and biochemistry due to their relatively high chemical inertness (in contrast to that of sodium particles - sodium is highly reactive even in the bulk form). The simplest analytic function often used to describe spectral properties of metals like gold or silver, results from the DrudeLorentz-Sommerfeld model supplemented by the interband electron transitions:

$$
\varepsilon_{m}(\omega)=\varepsilon_{i b}-\frac{\omega_{p}{ }^{2}}{\omega^{2}+i \gamma \omega} .
$$

$\omega_{p}$ is the plasmon frequency of a metal, $\gamma$ is the relaxation rate of free-electron movement accounting for ohmic losses, and $\varepsilon_{i b}$ is the phenomenological parameter describing the contribution of bound electrons to the polarizability: $\varepsilon_{i b}=1$ if the conduction band electrons only are responsible for the optical properties of a metal (the case of sodium). We accept the parameters of the Drude dielectric function $\varepsilon_{m}: \omega_{p}=9.01 \mathrm{eV}, \varepsilon_{i b}=9.84$, and $\gamma=0.072$ for gold and $\omega_{p}=9.1 \mathrm{eV}, \varepsilon_{i b}=3.7, \gamma=0.018 \mathrm{eV}$ for silver as those corresponding to one of the most widely available data sets of indices of refraction for pure metals [8]. For sodium, which is the best Drude free-electron metal, $\omega_{p}$ $=5.6 \mathrm{eV}, \varepsilon_{i b} \cong 1.06$, and $\gamma \cong 0.03 \mathrm{eV}$.

In our previous papers [3-7], multipolar LSP resonance frequencies $\omega_{l}^{\prime}(R)$ resulting from strict solutions of the dispersion relation, are given as a function of radius $R$ and plasmon polarity $(l=1,2,3, \ldots, 10)$. These data can be used directly in all basic problems and applications of LSP in which the well defined LSP resonance frequency is needed. These data can help to decide which metal must be used and how large the nanoparticle should be if embedded in the desired dielectric medium.

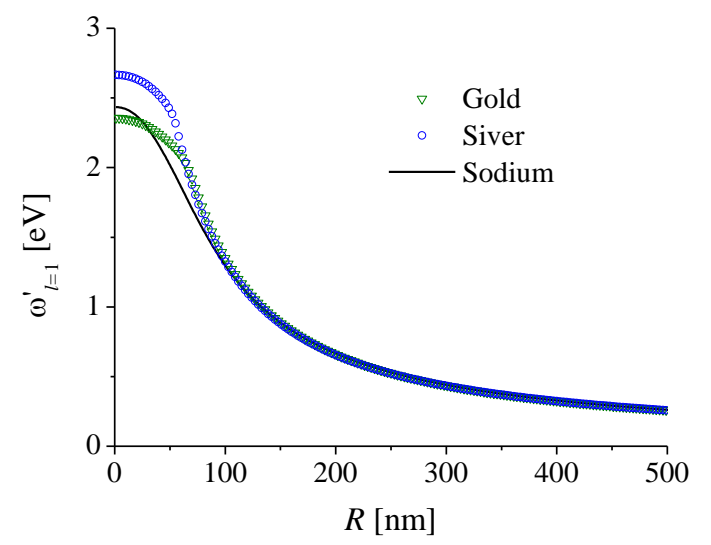

Fig. 1. Dipole plasmon resonance frequency of gold, silver and sodium nanospheres as a function of particle radius $R$.

For practical purposes, the dipole $(l=1)$ plasmon resonance frequency size dependence has been of importance till now, while manufacturing of larger nanoparticles of well controlled shape and dimensions still poses some problems. The well resolved spectral data about the size dependence of LSP were reported only for dipole plasmon resonance in nanoparticles of the size up to $R \simeq 75 \mathrm{~nm}$ for gold and $R \simeq 25 \mathrm{~nm}$ for silver $[1,2]$. Figure 1 shows the dependence of the dipole plasmon resonance frequency $\omega_{l=l}^{\prime}(R)$ for gold and silver nanospheres resulting from our strict solutions of the dispersion relation, Eq. (2). The index of refraction of the dielectric environment is $n_{d}=\sqrt{\varepsilon_{d}}=1.33$. The data for sodium are added to the figure as a reference illustrating LSP for the best Drude-type metal nanoparticles.

The aim of our present study was to give an even more comfortable tool allowing to predict the LPP resonance frequency as a function of size with the use of a very simple formula. We intended to find the algebraic expression which can be easily used by any person willing to calculate the plasmon resonance frequency for gold or silver nanoparticle of any size and obtain very similar results as those resulting from solving the full rigorous eigenvalue problem leading to the dispersion relation (2), without using any special functions of complex arguments and their derivatives. For that goal we use "normalized coordinates" in order to represent normalized LSP resonance frequency $\omega_{l}{ }^{1} / \omega_{p}$ versus normalized radius $R \omega_{p} / l c$ for the plasmon dipole mode $(l=1)$.

For the perfect Drude free-electron metal $\left(\varepsilon_{i b}=1\right.$ and $\left.\gamma=0\right)$, the dependence of $\omega_{l=l}^{\prime}(R) / \omega_{p}$ on $R \omega_{p} / l c$ should be independent on $\omega_{p}[9,10]$. Figure 2 proves this expectation for gold, silver and sodium nanospheres, if they are assumed to be made from the corresponding perfect free-electron metals. 


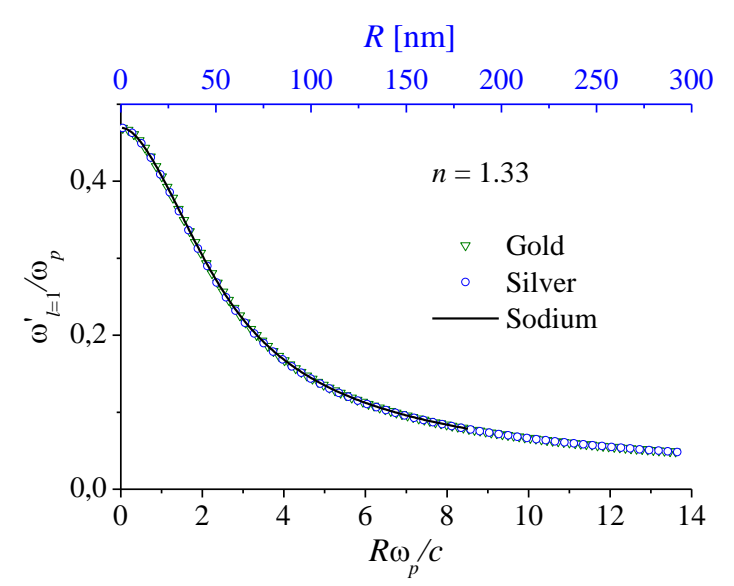

Fig. 2. Normalized plasmon oscillation frequencies as a function of normalized radius for idealized gold, silver and sodium nanospheres $\left(\varepsilon_{i b}=1, \gamma=0\right)$.

However, in real noble metals, interband transitions and ohmic losses influence their optical properties in an important manner. Also, the size dependence of $\omega_{l=l}^{\prime}(R) / \omega_{p}$ on $R \omega_{p} / c$ is not the same for these nanometals, as shown in Fig. 3. This is due to a difference in $\varepsilon_{i b}$ for real metals. Open symbols represent the dependence of a normalized plasmon oscillation frequency on a normalized radius for gold (green triangles) and silver (blue circles) resulting from the exact solutions presented in Fig. 1. The difference in $\omega_{l=l}^{\prime}(R) / \omega_{p}$ is very pronounced in the range of small $R \omega_{p} / c$ parameters and vanishes with increasing the normalised radius over 3 .

Our study shows that starting from the value of $R \omega_{p} / c=2.8$, the dependence of normalized plasmon oscillations frequencies on the normalized radius can be well described by a simple analytic Lorentz-type function, which is common for gold and silver nanoparticles:

$$
\frac{\omega_{l=1}^{\prime}}{\omega_{p}}=y_{0}+\frac{2 a}{\pi} \frac{w}{4\left(x-x_{c}\right)^{2}+w^{2}},
$$

for variable $x=R \omega_{p} / c$ and parameters: $y_{0}=0.02252$, $x_{c}=3.1921, w=0.49607$ and $a=95.57859$.

For a small range of $R \omega_{p} / c \leq 2.8$, the size dependence of a normalized plasmon frequency can be described by an analytical function:

$$
\frac{\omega_{l=1}^{\prime}}{\omega_{p}}=\frac{\omega_{0, l=1}^{\prime}}{\omega_{p}}+A x^{B},
$$

with the parameters $A=0.0059$ and $B=2.04312$ for gold and $A=0.02624$ and $B=1.64038$ for silver, and $\omega_{0, l=1}^{\prime}$ being a low radius limit of $\omega_{l=1}^{\prime}(R)$ [5]: $\omega_{0, l=1}^{\prime}=\omega_{p} /\left[\varepsilon_{i b}+2 \varepsilon_{d}\right]^{-1 / 2}$ (solid green and blue lines in Fig. 3, respectively).

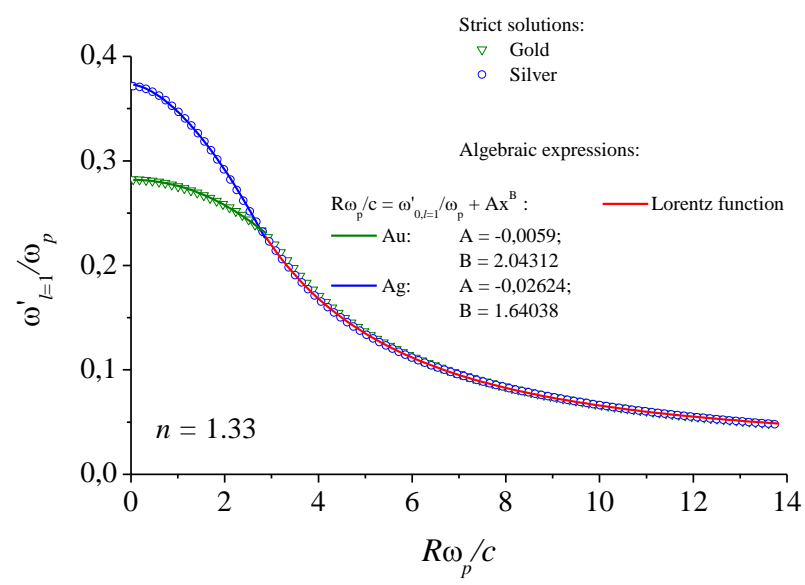

Fig. 3. Normalized plasmon oscillation frequencies as a function of normalized radius for real gold, silver and sodium nanosheres.

Figure 3 proves that the analytical Eqs. (4-5) with the coefficients, which are given above, can be used as a simple practical tool for predicting the size dependence of a dipole plasmon resonance frequency in gold and silver nanoparticles in a large size range without using a complex theory by using special functions of complex arguments and their derivatives.

The project was funded by the Ministry of Science and Higher Education, grant No N N202 126837 and by the National Science Centre on the basis of the decisions number DEC-2012/05/B/ST8/00087.

\section{References}

[1] K. Kolwas , A. Derkachova, S. Demianiuk, Comp. Mat. Sc. 35, 337 (2006). doi: 10.1016/j.commatsci.2004.09.063

[2] A. Derkachova, K. Kolwas, Eur. J. Phys. ST 144, 93 (2007). doi: 10.1140/epjst/e2007-00112-1

[3] K. Kolwas, A. Derkachova, M. Shopa, J. Quant. Spectros. Radiat. Transf. 110, 1490 (2009). doi: 10.1016/j.jqsrt.2009.03.020

[4] K. Kolwas and A. Derkachova, Opto-Electr. Rev. 18(4), 429 (2010). doi: 10.2478/s11772-010-0043-6

[5] K. Kolwas, A. Derkachova, J. Quant. Spectros. Radiat. Transf. 114, 45 (2013). doi: 10.2478/s11772-010-0043-6

[6] C. Sönnichsen, T. Franzl, T. Wilk, G. Von Plessen, J. Feldmann, New J. Phys. 4, 93 (2002). doi:10.1088/1367-2630/4/1/393

[7] H. Baida, P. Billaud, at all,. Nano Lett. 9, 3463 (2009). doi: 10.1021/n1901672b

[8] P.B. Johnson, R.W. Christy, Phys. Rev. B 6, 4370 (1972). http://link.aps.org/doi/10.1103/PhysRevB.6.4370

[9] T.V. Teperik, V.V. Popov, Phys. Rev. B 69, 155402 (2004). http://link.aps.org/doi/10.1103/PhysRevB.69.155402

[10] F. Forstmann, R.R. Gerhardts, J. Mod. Opt. 109, 291 (1982). 